\title{
A utilização do agulhamento seco nos músculos masseter e temporal para o tratamento da dor miofascial: Relato de caso
}

The use of dry needles in the masseter and temporal muscles for the treatment of myofascial pain:

\section{Case report}

El uso de agujas secas en los músculos masetero y temporal para el tratamiento del dolor

miofascial: Reporte de caso

Recebido: 31/05/2021 | Revisado: 06/06/2021 | Aceito: 06/06/2021 | Publicado: 20/06/2021

Gabriela De Melo Reis

ORCID: https://orcid.org/0000-0003-1560-0159

Faculdade Maria Milza, Brasil

E-mail: gabrielareis01@gmail.com

Amanda Feitoza da Silva

ORCID: https://orcid.org/0000-0002-4769-7517

Universidade Tiradentes, Brasil

E-mail: amandafeitoza@live.com

Juliana Campos Pinheiro

ORCID: https://orcid.org/0000-0001-5687-7635

Universidade Tiradentes, Brasil

E-mail: dra.julianacpinheiro@gmail.com

Denise Coelho de Souza

ORCID: https://orcid.org/0000-0002-4001-8737

Universidade Federal do Ceará, Brasil

E-mail: denisecoelho.sucesso@hotmail.com

Gleiciane Silva de Aguiar

ORCID: https://orcid.org/0000-0003-4491-5328

Faculdade Vale do Jaguaribe, Brasil

E-mail: gleicianeaguiarfisio@gmail.com

Ítalo Alexandrino Gonçalves Loiola

ORCID: https://orcid.org/0000-0003-1347-5294

Escola de Saúde Pública do Ceará, Brasil

E-mail: italoalexandrino@ hotmail.com

Túlio de Araújo Lucena

ORCID: https://orcid.org/0000-0002-2308-0432

Universidade Federal do Rio Grande do Norte, Brasil

E-mail: tuliolucena1999@gmail.com

Jabes Gennedyr da Cruz Lima

ORCID: https://orcid.org/0000-0001-7420-7686

Universidade Federal do Rio Grande do Norte, Brasil

E-mail: jabes.gennedyr@hotmail.com

Gabriel Coutinho Gonçalves

ORCID: https://orcid.org/0000-0002-5960-2976 Universidade Estadual do Ceará, Brasil E-mail: gabrielcoutinhoo@hotmail.com

Fellipe Moraes Pereira Figueiredo ORCID: https://orcid.org/0000-0003-3370-8681 Faculdade Maria Milza, Brasil E-mail: fellipempf@gmail.com

Anderson Lopes De Gois Santos

ORCID: https://orcid.org/0000-0003-3238-8962 Faculdade Maria Milza, Brasil

E-mail: andersonlopes@gmail.com

\section{Resumo}

$\mathrm{O}$ agulhamento seco tem como principal finalidade inativar os pontos-gatilhos miofasciais presentes nas bandas tensas musculares, que são áreas hipersensíveis à palpação que tem como sintoma estímulo de dor local e/ou dor referida. O presente estudo tem como objetivo fazer um relato caso sobre a utilização do agulhamento seco na síndrome da dor miofascial. Paciente, gênero feminino, queixava-se de disfunção temporomandibular e síndrome da dor miofascial, com presença de pontos-gatilhos nos músculos masseter e/ou temporal. Inicialmente foi realizada uma anamnese do tipo RDC/TMD, seguido de palpação dos respectivos músculos. A paciente marcou a intensidade da dor 
anteriormente e posteriormente à sessão do agulhamento seco, em uma escala visual analógica. Para analisar o efeito do agulhamento seco, os dados obtidos foram organizados a partir da comparação dos resultados atingidos com as técnicas realizadas. Verificou-se que o agulhamento seco foi eficaz no tratamento do ponto-gatilho miofascial, diminuindo consideravelmente a dor imediatamente após a sessão.

Palavras-chave: Síndrome da Disfunção da Articulação Temporomandibular; Acupuntura; Síndromes da Dor Miofascial.

\begin{abstract}
Dry needling has the main purpose of inactivating the myofascial trigger points present in the tense muscle bands, which are hypersensitive areas to palpation that have the stimulus of local pain and / or referred pain. The present study aims to report a case of the use of dry needling in myofascial pain syndrome. A female patient complained of temporomandibular disorder and myofascial pain syndrome, with the presence of trigger points in the masseter and / or temporalis muscles. Initially, an anamnesis of the RDC / TMD type was performed, followed by palpation of the respective muscles. The patient marked the intensity of the pain before and after the dry needling session, on a visual analog scale. To analyze the effect of dry needling, the data obtained were organized from the comparison of the results achieved with the techniques performed. It was found that dry needling was effective in treating the myofascial trigger point, considerably decreasing pain immediately after the session.
\end{abstract}

Keywords: Temporomandibular Joint Dysfunction Syndrome; Acupuncture; Myofascial Pain Syndromes.

\title{
Resumen
}

El objetivo principal de la punción seca es inactivar los puntos gatillo miofasciales presentes en las bandas de tensión muscular, que son áreas hipersensibles a la palpación y tienen como síntoma la estimulación del dolor local y / o dolor referido. El presente estudio tiene como objetivo reportar un caso de uso de punción seca en el síndrome de dolor miofascial. Paciente de sexo femenino que consulta por trastorno temporomandibular y síndrome de dolor miofascial, con presencia de puntos gatillo en masetero y / o músculos temporales. Inicialmente se realizó una anamnesis tipo RDC / TMD, seguida de palpación de los respectivos músculos. El paciente marcó la intensidad del dolor antes y después de la sesión de punción seca, en una escala visual analógica. Para analizar el efecto de la punción seca, los datos obtenidos se organizaron a partir de la comparación de los resultados obtenidos con las técnicas realizadas. Se encontró que la punción seca fue efectiva en el tratamiento del punto gatillo miofascial, disminuyendo considerablemente el dolor inmediatamente después de la sesión.

Palabras clave: Síndrome de la Disfunción de Articulación Temporomandibular; Acupuntura; Síndromes del Dolor Miofascial.

\section{Introdução}

Segundo a Academia Americana de Dor Orofacial, a disfunção temporomandibular (DTM) é um aglomerado de problemas clínicos relacionados à articulação temporomandibular (ATM), os músculos mastigatórios e suas demais estruturas relacionadas. Os sintomas mais comuns estão relacionados a presença de dor na face, cabeça, ATM, ouvido, zumbidos e vertigem. Os sinais se iniciam com sensibilidade à palpação muscular e da ATM, ruídos articulares e falta de controle ao movimentar a mandíbula (Venancio, 2005; Campos, 2007; Martins, 2015; Lopez-Martos, 2018; Melo, 2020).

No ponto de vista de Souza (2016), a fadiga das fibras musculares, muitas vezes ocasionada por sobrecarga, origina a formação dos pontos-gatilhos (PGM), que são áreas hipersensíveis à palpação, formadas por bandas tensas musculares, que quando pressionadas produzem um estímulo local ou de dor referida. Fatores como microtraumas, bruxismo do sono ou de vigília, onicofagia, tratamento odontológico prolongado, são condições que podem levar o individuo a desenvolver PGM.

Para Freitas (2011), os PGM podem ser desenvolvidos em qualquer pessoa independente de idade, profissão e gênero, porém acomete mais as mulheres e na faixa etária entre 20 e 50 anos. Essas características também são vistas nas DTM e na síndrome da dor miofascial (SDM), que são disfunções ligadas aos PGM nos músculos mastigatórios. A SDM é um estado não inflamatório típico e divergente dos outros distúrbios dolorosos dos tecidos moles, caracterizada pela presença de PGM. Ela se manifesta como uma dor regional e geralmente seguida de redução da flexibilidade e um aumento da tensão.

Não foi achado na literatura um acordo que estabelecesse a forma mais concreta e precisa para tratamento de pacientes que possuem DTM, pois apenas com uma correta avaliação de forma individual, o profissional vai poder julgar qual tratamento é mais indicado para cada caso. Existem inúmeras formas de tratamento na qual o profissional pode lançar mão 
para tratar indivíduos que apresentam DTM, como placas miorrelaxantes, contenção mandibular, toxina botulínica do tipo A, psicoterapia, tratamento invasivo por meio de cirurgia na ATM e agulhamento seco (Branco, 2008; Figueiredo, 2009; Fernández-Carnero, 2010; Costa, 2018). Diante do exposto, o presente estudo tem como objetivo fazer um relato caso sobre a utilização do agulhamento seco na síndrome da dor miofascial.

\section{Relato de Caso}

Paciente D.M.M, gênero feminino, 49 anos, leucoderma, compareceu à clínica odontológica relatando limitação da abertura de boca, fortes dores de cabeça e na face há aproximadamente 6 meses. Foi realizada a anamnese RDC/TMD, que continha perguntas voltadas para diagnóstico de disfunção temporomandibular e dor miofascial o que facilitava a padronização de estudos direcionados a DTM. Além disso, foram entregues duas Escalas de Análise Visual (EVA), onde a paciente marcou a sua dor de intensidade 9 no músculo masseter e intensidade 7 no músculo temporal. No exame físico, durante a palpação muscular, houve relato de dor familiar na região do masseter e do temporal do lado esquerdo. Durante o exame físico de palpação, foi encontrado um nódulo hiperritável que causava dor familiar (ponto gatilho), sendo que no músculo temporal esquerdo expandia para a região de molar esquerdo e no masseter esquerdo para o ouvido e região de sobrancelha esquerda. $\mathrm{O}$ diagnóstico da paciente foi de dor miofascial nos músculos masseter e temporal lado esquerdo com etiologia a definir.

A sessão do agulhamento seco se iniciou com a assepsia da pele com algodão embebido de álcool 70\% (Figura 1). Os pontos gatilho foram localizados por meio da palpação e prensados com os dedos indicador e médio, em seguida inseriu a agulha de acupuntura, estimulando em todas as direções do ponto gatilho de forma circular, intercalando com o repouso da agulha, foi realizado nos músculos Masseter (Figura 2) e Temporal (Figura 3).As sessões foram seguidas de massagem e alongamento dos músculos trabalhados e foram executadas por um cirurgião-dentista especialista capacitado.

Figura 1. Assepsia com algodão embebido com álcool $70 \%$.

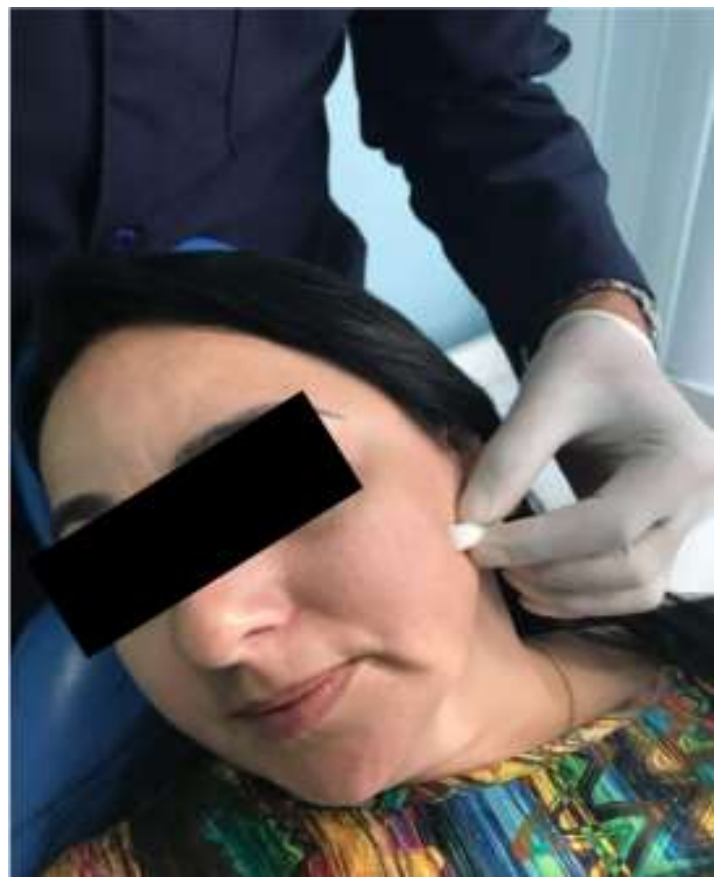

Fonte: Autores (2021). 
Research, Society and Development, v. 10, n. 7, e25510716650, 2021

(CC BY 4.0) | ISSN 2525-3409 | DOI: http://dx.doi.org/10.33448/rsd-v10i7.16650

Figura 2. Agulhamento seco no músculo masseter esquerdo.

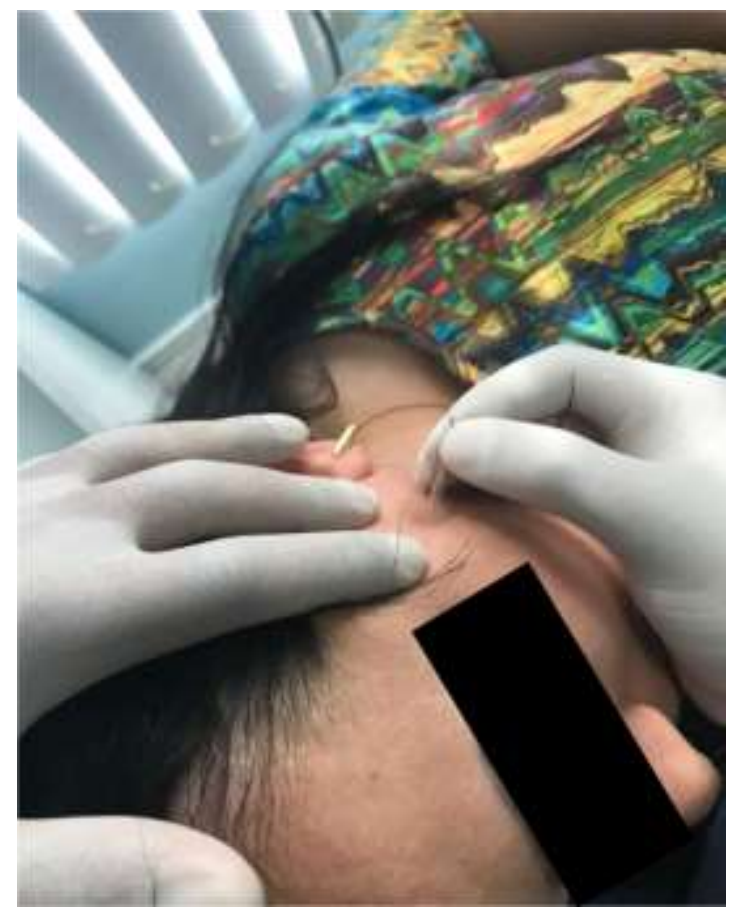

Fonte: Autores (2021).

Figura 3. Agulhamento seco no músculo temporal esquerdo.

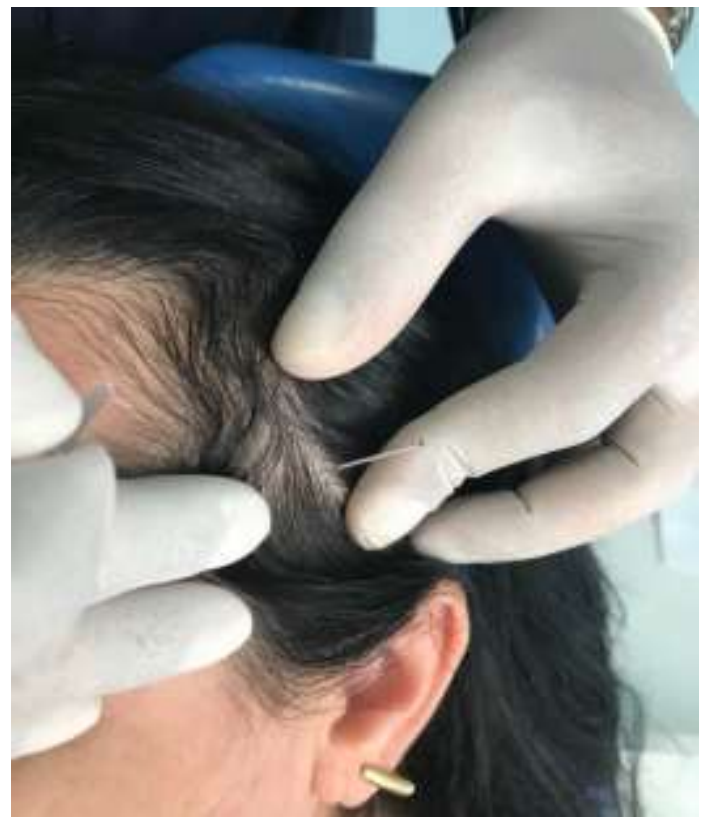

Fonte: Autores (2021).

Posteriormente ao tratamento utilizando o agulhamento seco, foi realizado novamente o EVA, para avaliar a intensidade da dor da paciente logo após a sessão de agulhamento seco. No momento da palpação de ambos os músculos (masseter e temporal) do lado esquerdo, a paciente relatou na EVA a intensidade de dor número zero, demonstrando um ótimo resultado clinico, com grande eficiência e alivio imediato com a utilização do agulhamento seco. 


\section{Discussão}

Os achados do presente relato de caso foram semelhantes ao estudo de Uemoto (2013), onde foram avaliados 21 pacientes, divididos em dois grupos: um grupo de tratamento com o agulhamento seco e outro grupo de tratamento com laser terapia, no qual se observou que tanto a utilização do agulhamento seco quanto a laser terapia, exibiram resultados eficazes e satisfatórios no tratamento da DTM.

O resultado se fez positivo devido ao principio neurofisiológico do agulhamento seco. Pois, ocorre uma inflamação na banda tensa muscular no momento que a agulha toca o ponto gatilho, gerando uma contração imediata seguido de um relaxamento total deste músculo (Felice, 2009; Tough, 2009; Srbely, 2010; Fernandes, 2011; Sposito, 2014). Essa inflamação faz com que o corpo responda, mandando células de defesa para o local a fim de controlar o processo inflamatório, agilizando assim o processo de reparação do músculo (Shirani, 2009; Bastos, 2017; Raeissadat, 2018). Como salienta Carvalho (2017), a inserção da agulha no PGM lesiona as placas motoras, desta forma, ocorre a desenervação axônica como consequência e induz a regeneração fisiológica, que acontece 7 a 10 dias após a sessão.

\section{Conclusão}

O desenvolvimento do presente estudo possibilitou uma análise da técnica do agulhamento seco, que consiste na palpação muscular para localizar o ponto-gatilho, inserção da agulha aproximadamente $1 \mathrm{~cm}$ e fazer movimentos de retirada parcial da agulha para abranger toda a extensão do ponto hipersensível. O agulhamento seco foi eficaz na diminuição da dor miofascial da paciente, mostrando-se como uma forma de tratamento eficiente para o controle dos pacientes que sofrem com esse problema.

\section{Referências}

Bastos, J. M. (2017). Disfunção temporomandibular: uma revisão de literatura sobre epidemiologia, sinais e sintomas e exame clínico. Revista da Saúde e Biotecnologia, 1(1):66-77.

Branco, R. S. (2008). Frequiência de relatos de parafunções nos subgrupos diagnósticos de DTM de acordo com os critérios diagnósticos para pesquisa em disfunções temporomandibulares (RDC/TMD). R Dental Press OrtodonOrtop Facial, 13(2):61-69.

Campos, J. (2007). Internal consistency and reproducibility of Portuguese version of research diagnostic criteria for temporomandibular disorders (RDC/TMDAxis II). Revista Brasileira de Fisioterapia, 11(60):451-459.

Carvalho, A. V. (2017). The use of dry needling in the treatment of cervical and masticatory myofascial pain. Revista Dor, 18(3): 255-260.

Felice, T. D. (2009). Recursos Fisioterapêuticos (Crioterapia e Termoterapia) na espasticidade: revisão de literatura. RevNeurocienc, 1(1):57-62.

Fernandes, E. H. (2011). Síndrome dolorosa miofascial em trabalhadores com LER/DORT. RevBrasMedTrab, 1(1):39-44.

Fernández-Carnero, J. (2010). Efeitos a curto prazo do agulhamento seco de pontos-gatilho miofasciais ativos no músculo masseter em pacientes com desordem temporomandibular. J OrofacPain, 24(1):106-112.

Figueiredo, V. M. G. (2009). Prevalência de sinais, sintomas e fatores associados em portadores de disfunção temporomandibular. Acta Scientiarum. Health Sciences, 31(2):159-163.

Freitas, D. G. (2011). Os efeitos da desativação dos pontos-gatilho miofasciais, da mobilização articular e do exercício de estabilização cervical em uma paciente com disfunção temporomandibular: um estudo de caso. Fisioter Mov, 24(1):33-38.

Lopez-Martos, R. (2018). Randomized, double-blind study comparing percutaneous electrolysis and dry needling for the management of temporomandibular myofascial pain. Medicina Oral Patología Oral y Cirugia Bucal, 1(1):454-462.

Martins, J. S. (2015). Avaliação do volume do disco articular da atm por meio de imagens de ressonância magnética usando um software de análise de imagem. Rev. Odontol. Univ. Cid. São Paulo, 125(118):118-125.

Melo, L. A. (2020). Manual Therapy in the Treatment of Myofascial Pain Related to Temporomandibular Disorders: A Systematic Review. J Oral Facial Pain Headache, 34(2), 141-148.

Raeissadat, S. A. (2018). Comparison of ozone and lidocaine injection efficacy vs dry needling in myofascial pain syndrome patients. Journal Of Pain Research, 11(1):1273-1279. 
Research, Society and Development, v. 10, n. 7, e25510716650, 2021

(CC BY 4.0) | ISSN 2525-3409 | DOI: http://dx.doi.org/10.33448/rsd-v10i7.16650

Shirani, A. M. (2009). Low-level laser therapy and myofacial pain dysfunction syndrome: a randomized controlled clinical trial. Lasers Med Sci, 24(5), 715720 .

Souza, L. M. (2016). A utilização da acupuntura no tratamento da disfunção da articulação temporomandibular: sugestão para um protocolo clínico. Ata de Ciências e Saúde, 2(5):112-128.

Sposito, M.M.M. (2014). Toxina Botulínica Tipo A no tratamento da dor miofascial relacionada aos músculos da mastigação. Acta Fisiatr, 21(3):152-157.

Srbely, J. Z. (2010). Dry needle stimulation of myofascial trigger points evokes segmental anti-nociceptive effects. J Rehabil Med, 42(5), 463-468.

Tough, E. A. (2009). Acupuncture and dry needling in the management of myofascial trigger point pain: a systematic review and meta-analysis of randomised controlled trials. Eur J Pain, 13(1), 3-10.

Uemoto, L. (2013). Laserterapia e agulhamento na desativação do ponto-gatilho miofascial. J Oral Sci, 55 (2):175-81.

Venancio, R. A.. (2005). Low intensity laser therapy in the treatment of temporomandibular disorders: a double-blind study. J Oral Rehabil, 32 (11), 800- 807. 